\title{
ENHANCING THE PERFORMANCE OF DIABETES PREDICTION USING TUNING OF HYPERPARAMETERS OF CLASSIFIERS ON IMBALANCED DATASET
}

\author{
Subhash Chandra Gupta \\ Research Scholar, Department of Computer Applications \\ VBS Purvanchal University, Jaunpur, India \\ csubhashgupta@gmail.com \\ Durgesh Kumar Singh \\ Research Scholar, Department of Computer Applications \\ VBS Purvanchal University, Jaunpur, India \\ durgeshsingh111@gmail.com \\ Noopur Goel \\ Assistant Professor, Department of Computer Applications \\ VBS Purvanchal University, Jaunpur, India \\ noopurt11@gmail.com \\ ORCID ID: 0000-0003-3351-376
}

\begin{abstract}
Background: The prediction ability of a classifier is important for a diabetes prediction model. The more correct prediction a classifier make, the better performance of the model will be. Although a number of researches has been done in this area, but still there are some scope to improve the performing capability of model. In this experimental work an effort is made to do it by applying these three method - identifying appropriate preprocessing act, perform oversampling to make balanced class dataset and tune the hyperparameter of classifiers to improve its performance.

Methods: In research experiment, four different prediction model is built on preprocessed, oversampled balanced class datasets which are created from PIMA diabetes dataset by different preprocessing methods. Each model uses hypertuned classifiers KNN, SVM, DT and random forest to classify samples in diabetic and non-diabetic category. The obtained results are stored and analyzed, and the best model is selected by considering $F 1$ score of classifiers of all prediction model.

Results: The results obtained from these models show that the highest F1score of classifiers of each model on dataset D1, D2, D3 and D4 are 88.52 \%, 88.79\%, $93.33 \%$ and $95.23 \%$ respectively, and it is achieved by random forest classifier for every model.

Conclusion: From the analysis of the results obtained from these models it is found that the best prediction model is based on dataset $\mathrm{D} 4$ which is created from the removal of outliers and rows having missing values during preprocessing.

Keywords: Diabetes mellitus; imbalanced dataset; over-sampling; SMOTE method; random forest; support vector machine; KNN.

\section{Introduction}

In human body, Glucose is one of the main sources of energy and found in a form of sugar in human blood. Sometimes the body is unable to extract glucose from blood and blood sugar level is increased due to it and the person comes under the diabetic category. Diabetes is a common disease which affects the human when pancreas does not produce enough insulin for the body or the body becomes unable to properly use the produced insulin or result of both. Insulin is a hormone that regulates blood sugar level in the body [1]. When this situation arises, it can cause many serious health problems, such as reduction in the capability of the body to utilize the energy present in the food. Diabetes leads the other health problem such as heart attack, kidney failure, blindness and
\end{abstract}


lower feet problems [2]. Healthy eating, regular physical exercise, maintaining normal weight and avoiding smoking are ways to prevent diabetes.

In human, diabetes are found in three types: Type 1 Diabetes, Type 2 Diabetes (Prediabetes) and Gestational diabetes. Type 1 Diabetes is known as Insulin-Dependent Diabetes Mellitus (IDDM) [3] and also called as Juvenile-onset diabetes [4]. Diabetes is the result of an autoimmune condition of the body in which beta cells of the pancreas are attacked, and it destroyed. The damaged pancreas does not produce enough insulin and in result the level of glucose increases in blood and crossed the normal range [5], [6]. In Type 2 diabetes, body cells becomes insulin resistance and unable to consume insulin produced by pancreas. This situation stimulates the pancreas to produce more insulin. Due to over functioning, the pancreas affected badly and stop the production of insulin, which increases the glucose level in body [7]. Type 2 diabetes is also called as Non-Insulin-Dependent Diabetes Mellitus (NIDDM) or Adult-Onset Diabetes [4]. It regularly happens in grown-ups. The factors causing type-2 diabetes are hereditary, obesity family history, overweight body, smoking and lack of exercise [8], [9]. Gestational diabetes appears only in women during their pregnancy and disappears after their deliveries. High glucose level in blood is diagnosed during pregnancy in the women whose medical history shows that they are not affected by diabetes earlier [10]. After delivery, blood sugar level returns to normal level, but it increases the chance of type-2 diabetes [11]. Although it may happen at any stage of pregnancy but second or third month is common for it.

Prediction is one of the major applications of data mining in different areas. In health sector, disease prediction is one of them and may be used to save the life of thousands of people. Patient's Medical history, test reports, electronic clinical devices of hospitals produces lots of data for medical research. These datasets can be used to extract needed information which makes an intelligent diagnosis system for a specified disease. AIDS, Cancer, Tuberculosis and diabetes can be diagnosed and predicted using data mining techniques [12]. A disease prediction model is developed to correctly classify given samples in positive or negative results. The correct diagnosis of disease of a patient increases the chances of his recovery. The better the performances of a model, the less chance of making errors by it. Although, a number of researches performed in past for diabetes prediction model, but there is still a scope of improvements about prediction/ classification of disease. The main objective of the research paper is to improve the prediction capability of the model by enhancing the performances of classifiers used in the model. It is to be performed by these three ways.

- By selecting appropriate preprocessing method: A dataset may contain missing values and/ or outliers, and they also might affect the performance of prediction model. A proper preprocessing of dataset may improve the performance of classifiers. An analysis is performed to identify the most effective preprocessing method for input diabetes dataset by which the best result obtained.

- Making balance class dataset by oversampling: Input PIMA dataset is an imbalanced dataset and to get better result, it must be converted to a balanced class dataset. Since dataset size is small, so oversampling is performed to synthesized minority class samples.

- Hypertuning of classifiers: A classifier's performance is strongly affected by the value of its hyperparameters. An effort is made to tune some hyperparameters of classifiers to improve the prediction ability of classifiers of model.

The research paper is divided into nine different sections. The first and current section is the introduction which discuss the diabetes and its global prevalence and objective of paper. Section 2 briefly discusses past researches and their outcomes. The section 3 is about machine learning algorithms, preprocessing methods and handling of imbalanced class dataset, which have used in this prediction model. Discussion about working methodology of the model is made in Section 4. Section 5 covers the result and discussion for all four dataset models. Here, a comparative analysis of performances of all models from the point of each metrics is done. It also focuses on emphasis of selection of F1 score as the main metrics for examination and selection of the best dataset model for detail discussion. The selected dataset models and its classifier's performances are discussed in detail in Section 6. Section 7 is about the effect of preprocessing methods on prediction of Diabetes using different classifiers and dataset models. Sections 8 and 9 are the findings of research examination and conclusion respectively.

\section{Literature Review}

N. Sneha et al. [1] have designed a prediction model whose aim was to focus on selection of optimal attributes and made a predictive analysis for early detection of Diabetes Miletus disease. They had worked on a diabetes dataset which contains record of 2500 patients. Each patient's record data comprised about 15 different test parameters. Feature selection methods has been applied to remove irrelevant attributes from dataset. Further they have applied SVM, KNN, Naive Bayes, Decision tree and Random forest classifiers to test their prediction model. From developed model they have achieved the best accuracy of $82.2 \%$ for Naive Bayes amongst other classifiers, although an enhancement scope may possible from Random forest algorithm. R J. Steffi et al. [4] has worked on PIMA diabetes dataset and used five classification algorithms for their prediction model. They have made a comparative analysis between the performances of ANN, Naive Bayes, Logistic Regression, C5.0 and SVM 
classifiers and achieved their best accuracy of 74.67 using Logistic regression. They have also made a comparison between the times taken by all classifier. It is shown by their research that C5.0 and ANN has taken minimum and maximum time respectively to declare results.

For diabetes prediction, Aiswarya Iyer et al. [7] worked on PIMA Indian Diabetes Dataset and used Naive Bayes and J48 Decision tree. They have used WEKA tool to implement the model. Feature selection and normalization of dataset was performed and achieved $79.56 \%$ accuracy level from Naive Bayes classifier for diabetes prediction. In [13], A. Amina et al selected PIMA Indian Diabetes dataset for their research work. Numerical values were converted in categorized value using WEKA tools and after it NB, Decision tree and KNN classifiers were applied. The obtained result was validated by cross validation and got best accuracy of $75.65 \%$ using Decision tree classifier. Since KNN works better on numerical data due to the concept of similarity measures, so an improvement in performance of KNN classifier may possible.

In this paper [14], M. Francesco et al. proposed a method that was able to classify diabetes patients using the set of characteristics defined by World Health Organization criteria. They have scored the highest precision 77\% and $77.5 \%$ recall using the Hoeffding Tree algorithm applying on real-time data. In [15], N. Nai-Arun et al. developed a model based on well-known classification algorithm such as decision tree, Logistic regression, Naïve Bayes, Artificial Neural Network and random forest and check its robustness by using Bagging and boosting techniques. Research work was performed on medical history of people taken from Sawanpracharak Regional Hospital during $2012-2013$. Best accuracy is $85.56 \%$ which is achieved by Random Forest. Zhang et al [16] have worked on diabetes dataset to study the impact of psychosocial and contextual barriers on Type 1 diabetes in adolescents. An Ecological momentary assessment (EMA) system using mobile technology was developed by them to assess the psychological process, subjective experience and contextual impact on self-management decision-making for diabetes in life. To achieve this goal, the model was trained with a machine learning algorithm. Data was collected using the MyDay Mobile app, and machine learning classifiers made prediction about two self-management behaviours for diabetes control. They had achieved $74 \%$ accuracy level for their work. Although diabetes is incurable, but a proper managed life-style may avoided its threats. Elliot B. Sloane et al. [17] have proposed a model based on a cloud-based mobile application which makes a diabetes monitoring system that integrates the diabetic patient, physician and diabetes coaches. It would properly monitor patient's life-style information and intervened if critical.

The above researchers and some more have tried to find the best performances in their research work by applying different classifiers and also achieved their best. But there is still a scope of improvement in performance of classifiers. For a disease prediction system, the performance of a classifier should be much better. So the objective of this research work is the enhancement of performance of different classifiers. Works of some researchers are listed below in Table 1.

Table 1: Past researches done by different researchers

\begin{tabular}{|c|c|c|}
\hline Reference & Author \& Year & Classification Algorithm (Accuracy in \%) \\
\hline [1] & N. Sneha and Tarun Gangil (2019) & $\begin{array}{l}\text { KNN (63.04), SVM(77.73), NB (73.48), Decision Tree ( 73.18) and } \\
\text { Random Forest (75.39) }\end{array}$ \\
\hline [4] & $\begin{array}{l}\text { J. Steffi , R. Balasubramanian and K. Arvind Kumar } \\
\text { (2018) }\end{array}$ & $\begin{array}{l}\text { Naïve Bayes (73.57), Logistic Regression (74.67) ,Decision Tree } \\
(\text { C4.5) }(74.63), \text { SVM (72.17) and ANN (72.29) }\end{array}$ \\
\hline [7] & I. Aiswarya, S. Jeyalatha and S. Ronak (2015) & Decision tree (J48) and Naïve Bayes \\
\hline$[10]$ & R. Sengamuthu, R. Abirami , D. Karthik (2018) & Naive Bayes, Decision Tree (J48),PLS -LDA, SVM , BLR ,KNN \\
\hline$[13]$ & $\begin{array}{l}\text { A. Amina, A. Yasir, A. Muhammad, and Z. } \\
\text { Khurram (2018) }\end{array}$ & KNN (65.19), Naïve Bayes (71.74) and Decision Tree (75.65) \\
\hline$[14]$ & M. Francesco , N. Vittoria and S. Antonella (2017) & $\begin{array}{l}\text { Decision Tree (J48), Multilayer Perceptron , Hoeffding Tree, Naïve } \\
\text { Bayes and Random Forest }\end{array}$ \\
\hline$[15]$ & N. Nongyao , M. Rungruttikarn (2015) & $\begin{array}{l}\text { Decision tree (85.09), Artificial neural network (84.532), logistic } \\
\text { regression }(82.308) \text {, random forest }(85.56) \text {, Bagging and Boosting }\end{array}$ \\
\hline$[18]$ & Kumar P. Suresh, Umatejaswi V. (2018) & Random forest, Naive Bayes, C4.5 and simple logistic \\
\hline$[19]$ & S. Hina, A. Shaikn, Sattar (2017) & $\begin{array}{l}\text { Naïve Bayes (76.3), ZeroR (67.5), Decision tree J48 (75.3) Random } \\
\text { Forest (79.2), Multilayer Perception (81.82) and Logistic Regression } \\
(79.2)\end{array}$ \\
\hline [12] & Tahani Daghistani, Riyad Alshammari (2016) & The SOM algorithm, random forest and C4.5 decision tree \\
\hline [20] & Lukmanto, Rian Budi and E. (2015) & Fuzzy Hierarchical model \\
\hline
\end{tabular}




\section{Theoretical support of Experimental Analysis}

\subsection{Preprocessing}

In machine learning, data preprocessing is the step to transform dataset in such a manner that machine learning techniques can easily interpret it. Data obtained from real world may be incomplete, inconsistent, missing values for some features and contains many errors or outliers. To solve such type of issues, data preprocessing is a wellknown method. Data preprocessing is the set of techniques which transform the raw data of a dataset into an understandable format. The most common errors in dataset are the incomplete samples, it means some samples have missing values or unknown values. It happens when data gathering process applied some faulty collection process or desired data is unavailable during collection process. Missing data can be handled in different ways as ignoring the tuples, deleting the rows/columns having missing values and filling the missing values.

- Ignoring tuples: In this method, no operation is performed, and it is suitable when the dataset is quite large and most of the columns have missing values for a tuple.

- Deleting missing values rows: In this method, the rows having missing values are removed from the dataset. It is applicable when dataset has enough samples. But it may affect the classifier's performance due to biasness and loss of information in dataset.

- Replacing with Mean, Median or Mode: This method is applied for features which has numeric values. The missing values of these features are replaced either by the mean, median or mode of the corresponding features or by the most preferable values. It is a statistical approach to handle missing values and sometimes produces better results as compared to the removal of rows having missing values. Classifiers show different behaviours for various methods of filling missing values [21].

\subsection{Handling of Imbalanced Dataset}

A dataset is considered as imbalanced class when there is a huge gap amongst the number of different classes in the dataset. For a binary classification problem, a dataset is balanced if the number of both classes are approximate equal, otherwise it is considered as an imbalanced dataset. Being input dataset balanced is rare in machine learning classification problem. A little imbalance in dataset is not a problem, but a high imbalanced class dataset may cause a significant degradation in performance and need some modification to handle it.

Only accuracy cannot be a good metrics for such type of imbalanced data. In a binary classification problem, if most of the data points are belongs to true class its accuracy will be always high, even though classifiers used in the model have not learnt anything about the problem. Most of the metrics are calculated only from TP, FP and FN. Imbalanced class problem can be solved by either changing the performance metrics such as F1-score, True Positive Rate, False Positive rate or using Imblearn Techniques. In this experimental analysis, F1-score and Imblearn Techniques (Resampling Techniques) are used to handle this problem. In Imblearn Techniques [22], a new dataset is generated from input dataset that has an equal ratio of both classes. The imbalance problem is treated by either Oversampling (Upsampling) or Downsampling. To make equal ratio of both classes, the minority class samples are regenerated in Upsampling and majority class samples are reduced in Downsampling. The diabetes dataset is an imbalanced binary class dataset and most of the samples are of negative samples. To handle imbalanced PIMA diabetes dataset, Over-sampling method is applied in this research examination. In oversampling, synthetic samples are produced by taking random samples of the attributes from instances of minority class.

SMOTE Techniques: SMOTE (Synthetic Minority Oversampling Technique) is a popular method to handle imbalanced dataset by oversampling of minority class. It synthesized new samples from existing minority samples, although the synthesized samples do not add any new information. Using the distance function in K-nearest neighbors, SMOTE algorithm selects two or more instances of a minor class and synthesized a new instance of the same class by randomly changing attributes of neighbouring instances [23], [24]. The dataset is reconstructed after the oversampling process.

\subsection{Machine Learning classification Algorithm}

Prediction is one of the major applications of machine learning in different area. In health sector, disease prediction is one of them. Patient's Medical history, test reports, electronic clinical devices of hospitals produced lots of data for medical research. These datasets can be used to extract useful information which makes a diagnosis for a specified disease. AIDS, Cancer, Tuberculosis and diabetes can be diagnosed and predicted using data mining techniques. In this paper, research is focused on the prediction of diabetes disease in pregnant women considering the different attributes in dataset. 


\subsubsection{K- Nearest Neighbor (KNN)}

K-Nearest Neighbor technique is a supervised machine learning algorithm applied for classification and regression problem. It is non-parametric because it does not have any assumptions about the distribution of data. A classification relation is identified by the attributes in training dataset. K- Nearest neighbors uses similarity measures (e.g. distance functions - Euclidean, Manhattan and Minkowski) to classify new objects [25][26]. Distance metrics are used to assign weights to the contribution of neighbors. Classification of the object is performed by the weights given by the $\mathrm{k}$ neighbors. Here, $\mathrm{k}$ defines the amount of data items selected from the nearest neighbors. The object is classified into the groups of its neighbors from which it is the closer [1][27]. Euclidean distance between point $\mathrm{X}$ and $\mathrm{Y}$ is calculated by the given equation-

$$
D(X, Y)=\sqrt{\sum_{i=1}^{n}\left(x_{i}-y_{i}\right)^{2}}
$$

\subsubsection{Decision Tree}

Decision tree is applied for the prediction, classification and regression problem. The model developed by decision tree is in the form of tree structure whose topmost node is the root node, internal node shows the attribute and branch node specifies the applied decision rule and result is shown by leaf node [13]. Learning ability is developed by the set of if-then-else decision rule which is based on some attribute and partition the dataset into two are more sub-datasets. Partitioning process is performed in recursive manner, showing the associated decision rule [7], [10]. The outcome of decision tree is in the form of a tree with decision and leaf nodes. Classification or decision is represented by leaf nodes. A decision node has two or more branches. The depth of tree shows the complexity of decision rule and fitting of the model. The best predictor attribute is represented by root node [4]. A decision tree, which is a supervised learning method, are easy to understand and interpret due to its flowchart like visualization. Decision tree can be applied for the categorical and numerical data sets.

\subsubsection{Random Forest}

The random forest algorithm is a supervised classification algorithm that randomly built a number of decision trees, where each decision tree represents a classifier. Each classifier makes prediction about one target class out of all the target classes. Prediction of outcome is based on majority voting of the target class predicted by multiple classifiers. Since it creates a number of classifiers, so it is also called as Ensemble classification method. Random forest algorithm gives the high accuracy results due to the higher number of decision trees it forms [1].

Random forest is slightly different from decision tree. In decision tree, all features or set of interested features of entire dataset is used to build a tree while in random forest multiple decision trees are formed by random selection of observations or rows and specific features and finally make average of them. Random forest is applied for classification and regression problems. It produces better result in comparison to other classification algorithms because it reduces the overfitting problem and is used to identify the most important features in the training dataset.

\subsubsection{Support Vector Machine}

Support Vector Machine (SVM) algorithm has been developed by Corinna Cortes and Vladimir Vapnik, and it is a supervised learning method. SVM is used for data analysis, pattern matching, and classification and regression analysis [4]. The standard SVM is applied for non-probabilistic binary classification data in which data prediction of outcome comes under the category of two class labels that is either 'Yes' or 'No'. The SVM classifier builds a model using training dataset samples and then uses this model to make prediction for test dataset samples. Conceptually, SVM puts dataset samples in a two - dimensional or infinite dimensional space and constructs a hyperplane or set of hyperplanes to separate the samples into two or more categories by as wide and clear margin as possible. Then the new example is matched with the samples of same space and predicts the side of category from which they belongs.

The hyperplane used for any dimensional data space is a linear data separator that classifies the data into two groups with suitable margin. The data space can be divided by infinite number of hyperplanes, but the best hyperplane is the one which maximizes the margin. The distance between the hyperplane and support vectors (closed data points) is called margin. The more margins a hyperplane have, the less error will be in data prediction [1].

\subsection{Hyperparameter Tuning}

Each classifier has a number of parameters whose values may vary to get better result for different dataset in various situation. In machine learning, values are passed to these parameters to set the behaviour of the classifier 
to specific datasets. These values are called hyperparameters and passed by the practitioners during the model configuration. It is a very difficult task to identify hyperparameters and its correct values for a classifier on a given dataset. The classifiers used in research, and their hyperparameters, are given below in table 2-

Table 2. Classifiers and theirs Tuned Hyperparameters

\begin{tabular}{|c|c|c|}
\hline Classifiers & Tuned Hyperparameters & Range / values \\
\hline \multirow{4}{*}{ KNN } & algorithm & 'auto','ball_tree', 'kd_tree', 'brute' \\
\hline & metric & 'euclidean' , 'minkowski' \\
\hline & n_neighbors & Range 1 to 70 \\
\hline & leaf_size & 30 \\
\hline \multirow{4}{*}{ Decision Tree classifier } & criterion & 'gini', 'entropy' \\
\hline & maximum depth & Range 1 to 30 \\
\hline & random state & Range 1 to 50 \\
\hline & minimum_sample_size & Range 1 to 30 \\
\hline \multirow{5}{*}{ Random Forest } & n_estimators & Range 1 to 50 \\
\hline & criterion & 'gini', 'entropy’ \\
\hline & random state & 42 \\
\hline & max_features & 'auto' \\
\hline & min_samples_leaf & Range $(1,50)$ \\
\hline \multirow[t]{4}{*}{ Support Vector Machine } & kernel & 'rbf', 'linear' , 'polynomial' \\
\hline & C & Range 1 to 100 \\
\hline & gamma & scale' \\
\hline & degree & 1 to 3 \\
\hline
\end{tabular}

\subsection{Performance Evaluators of Prediction Model}

\subsubsection{Performance Metrics}

The performance of classifiers is the most important for any predictive model, especially when the model is built for the disease detection. A wrong diagnosis may have to pay a heavy cost of life. Hence, the selection of a performance metrics plays a very crucial role in a disease diagnosis system. In data analysis system, there are a number of performance metrics such as F1score, accuracy, recall, precision and specificity. The Confusion matrix of a classifier provides necessary information to calculate the value of these metrics. It forms a base matrix which shows the number of predicted results and categorizes it into TP, FP, FN and TN [27][28]. The metrics precision, sensitivity, specificity, accuracy and F1score are calculated as follows:

$$
\begin{aligned}
& \text { Precision }=\frac{T P}{(T P+F P)} \\
& \text { Sensitivity }=\frac{T P}{(T P+F N)} \\
& \text { Specificity }=\frac{T N}{(T N+F P)} \\
& \text { Accuracy }=\frac{(T P+T N)}{(T P+T N+F P+F N)} \\
& \text { F1 score }=2 * \frac{(\text { precision } * \text { recall })}{(\text { precision }+ \text { recall })}
\end{aligned}
$$

\subsubsection{Selection of F1 Score as the best Metrics for Predictive Model}

High accuracy of a classifier does not ensure that the classifier correctly predicts most of the positive results. A high accuracy may be the result of more number of correct predictions of true-negative cases. So, only high accuracy of a classifier cannot be considered as a good measure for a classification algorithm. In a predictive analysis system, a wrong prediction may be a false-negatives or false-positives. The cost of these two wrong predictions may vary from one system to other system. In one system, a false-negative result may incur more cost from a false-positive result. For example in a sick detection system such as Diabetic prediction, classifier cannot afford a wrong prediction about a patient which is actually diabetic (TRUE- POSITIVE) and is predicted as nondiabetic (FALSE-NEGATIVE). So we need a model in which the chance of false-positives and false-negatives is 
less. In other words, its precision should be high since number of false-positives is less, similarly recall should be also high because it shows lower number of false-negatives. A high-precision and high recall of classifier ensures that it predicts less number of False-Positive and False-Negative results. In case of high false-positives cost, precision will be good measure and recall is for high false-negative cost.

Accuracy is a good measure if cost of false positives and false negatives both are the same. When it is different, then precision and sensitivity both are considered. In such situation, F1score is a better performance metrics among these. F1 score metric is the weighted average of precision and sensitivity, considering both false positives and false negatives. The higher value of F1 score shows the better performance of predictive model. So, F1 Score is considered as the main metrics to evaluate the performance of different classifiers for these datasets.

In case of an imbalanced dataset, F1score is not a good measure since it is biased to positive samples and considers only TP, FP and FN of confusion matrix of a classifier. In these circumstances, either dataset has to make balanced or used some other metrics such as MCC, macro f1score or micro flscore. Matthews Correlation Coefficient (MCC) is calculated from all components (TP, FP, FN and TN) of the confusion matrix. Its values lie between -1 and +1 . A +1 score shows that a model is perfect, while -1 score shows a poor model. Macro flscore is the average of flscores of all classes and gives equal weight to each class, while micro flscore gives equal weight to all samples of dataset. If dataset is balanced and contains equal number of samples of each class then its macro f1score and micro f1 score will always be equal [22]. Since PIMA diabetes dataset is an imbalanced dataset, so over-sampling has been done by SMOTE method to make balanced diabetes dataset. And F1score is considered as the main metrics for the research analysis.

\section{Work Flow of Proposed Model (Methodology)}

The prediction model is implemented in python language and used PIMA diabetes dataset as the main dataset. The prediction model creates four sub-models and these are based on different dataset versions which are created by different preprocessing methods on actual dataset. The goal of model is to classify the samples in diabetic and non-diabetic category and focused on the effect of preprocessing methods on the performances of classifier's prediction ability. The function of proposed model is performed in following major steps:

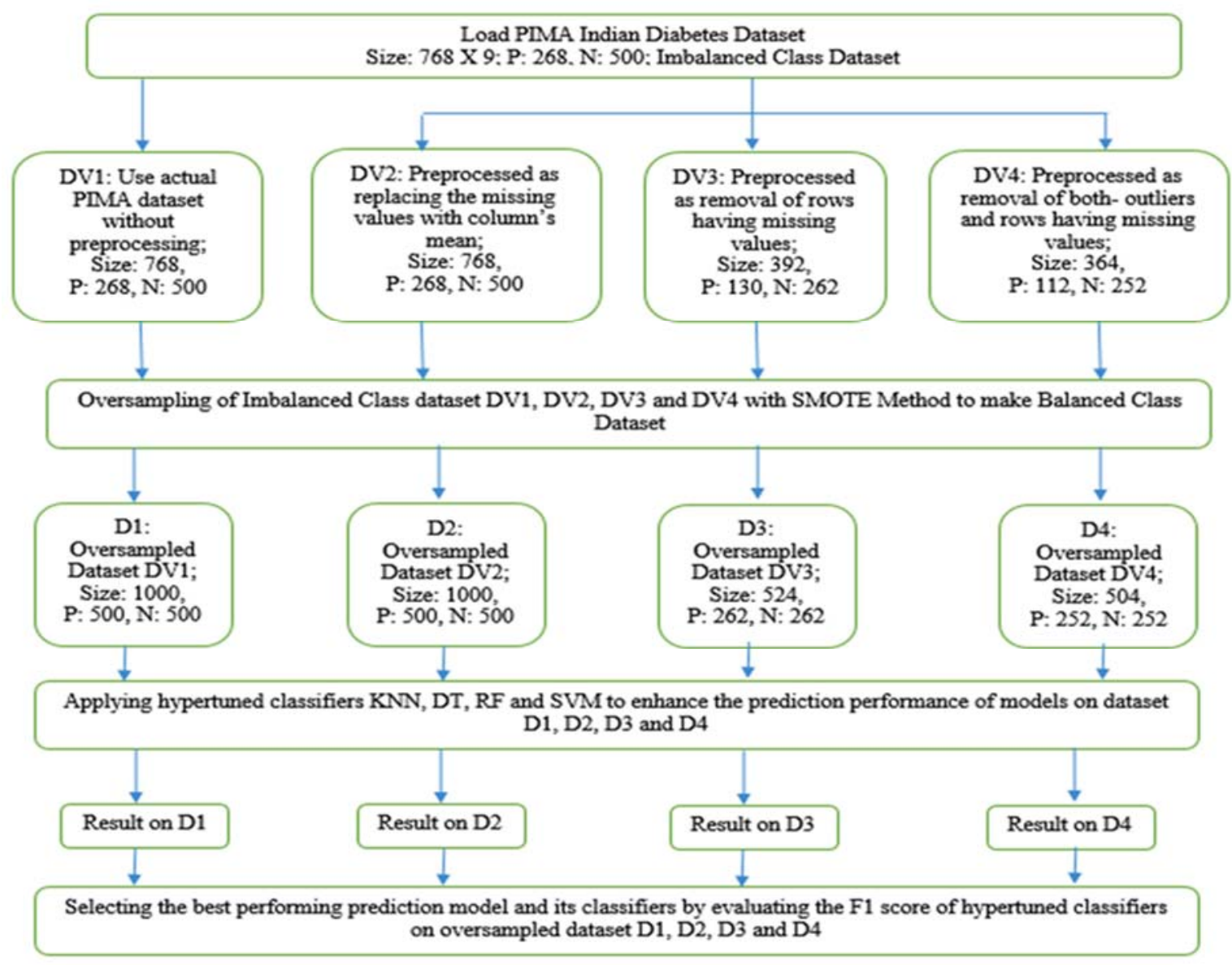

Fig 1: Work flow of Proposed Model of Diabetes Prediction 


\subsection{Load PIMA Indian diabetes dataset}

The Pima Indian diabetes dataset is the collection of test data related to women of Phoenix town situated in Arizona, state of the United States of America. The Pima are a native community living in Arizona State. Due to the change of their diets which caused them a victim of the highest prevalence of type 2 diabetes, they have been a subject of research studies. The source of PIMA dataset [29] is UCI Machine Learning Repository and downloaded from it. In this Dataset, test data of 768 patients (500 negative samples and 268 positive samples) are stored and each record stored 8 parameters according to their diagnosis. Dataset contains a class attribute "Outcome" which shows the result of test and in binary form $(0,1)$. A 1 value of class parameter shows the positive diagnosis of diabetes, while a 0 value represents negative result of diagnosis.

\subsection{PIMA Dataset Preprocessing}

During Data collection process, sometimes data of some particular attributes are unavailable and hence they have either 0 values or no values. Some unrealistic data are also stored due to typographic errors or other reasons. Outliers may also present in dataset. In dataset there are six attributes which contains zero values. The occurrences of zero in pregnancy attribute is 111 , glucose 5, BloodPressure 35 , skinthickness 227, insulin 374 and BMI has 11 zero values. These values may affect classifier's performance in adverse manner. To build a robust prediction model input dataset must be consistent. Unknown values may be filled with some appropriate values or remove the records which has missing values. Data preprocessing is the steps to remove such type of noise from data set and filling missing values. Here Input dataset is preprocessed by four preprocessing method and converted into four different datasets. The details of these four datasets are given in Table 3.

Table 3. Shape of different version of PIMA dataset preprocessed by different methods

\begin{tabular}{|c|c|c|c|c|c|}
\hline \multirow[t]{2}{*}{ Dataset } & \multirow{2}{*}{$\begin{array}{l}\text { Shape of } \\
\text { Dataset }\end{array}$} & \multirow{2}{*}{$\begin{array}{l}\text { No. of (+ve } \\
\text { /-ve) } \\
\text { Samples }\end{array}$} & \multirow[t]{2}{*}{ Preprocessing Activities } & \multicolumn{2}{|c|}{ After Over-Sampling of datasets } \\
\hline & & & & size & $\begin{array}{l}\text { number of (+ve /- } \\
\text { ve) Samples }\end{array}$ \\
\hline $\begin{array}{l}\text { D1- Actual Pima Diabetes } \\
\text { Dataset }\end{array}$ & $768 \times 9$ & $268 / 500$ & $\begin{array}{l}\text { Preprocessing is not } \\
\text { performed }\end{array}$ & $1000 \times 9$ & $500 / 500$ \\
\hline $\begin{array}{l}\text { D2- Dataset filling missing } \\
\text { values with column's mean } \\
\text { value }\end{array}$ & $768 \times 9$ & $268 / 500$ & $\begin{array}{l}\text { Fill all unknown values by } \\
\text { corresponding column's mean } \\
\text { value }\end{array}$ & $1000 \times 9$ & $500 / 500$ \\
\hline $\begin{array}{l}\text { D3- Dataset removing rows } \\
\text { having unknown values }\end{array}$ & $393 \times 9$ & $130 / 263$ & $\begin{array}{l}\text { Remove all rows which have } \\
\text { unknown value }\end{array}$ & $524 \times 9$ & $263 / 263$ \\
\hline $\begin{array}{l}\text { D4- Dataset removing } \\
\text { outliers and rows having } \\
\text { missing values }\end{array}$ & $364 \times 9$ & $112 / 252$ & $\begin{array}{l}\text { Remove rows having missing } \\
\text { values and outliers }\end{array}$ & $504 \times 9$ & $252 / 252$ \\
\hline
\end{tabular}

\subsection{Handling of Imbalanced Dataset}

In python, SMOTE (Synthetic Minority Over-sampling Techniques) is the most popular oversampling technique. Using a distance function, SMOTE algorithm selects two or more instances of a minor class and synthesized a new instance of the same class by randomly changing attributes of neighbouring instances [24] .

\subsection{Classification Algorithm with Hyperparameter Tuning and store results}

Each dataset is divided in ratio of 80:20 into training and test dataset. Training dataset is used to build the model, while test dataset is used for testing purpose. Major classification algorithm such as KNN, SVM, decision tree and random forest are applied for model building and testing process. Each classifier's hyperparameters are tuned to improve its performance. The classifiers used in research and their hyperparameters are given in table 2.

\subsection{Make Comparative Analysis of obtained result}

Each dataset model produces its result obtained from different classifiers. Performance of every classifier is measured in accuracy, precision, sensitivity, specificity and F1 score. For this prediction model, F1score is the main metrics. A comparative analysis is performed on these results of all versions of dataset and selects the best performing dataset model and its best classifiers. The best dataset model is that who performs better on F1 score and accuracy.

\section{Result and Discussion}

\subsection{Application of Classification Algorithm on Different Dataset Models of PIMA Diabetes}

In experimental analysis, KNN, SVM, Random Forest and Decision tree classifiers have been applied for prediction models on all version of dataset and their performances are analyzed to identify the best prediction model, its classifier and the preprocessing method by which a better result is found. All datasets are divided in 80:20 ratios in training and test dataset. Samples of training dataset are applied to build models and test dataset 
are applied to check the performance of model. The results obtained from different classifiers for prediction models are shown in Fig-2 and tabulated in table-4. The Accuracy, specificity, sensitivity and precision of classifiers of models (according to dataset) is shown in Fig-2 while F1score of models is shown in Fig-3.

The maximum precision of $100 \%$ is obtained by random forest in dataset D4. It shows that all positive predictions made by it are actually correct. Minimum precision of $73.45 \%$ is achieved by SVM classifier with linear kernel model on dataset D1. Decision tree classifier shows the best precision of $91.91 \%$ for model on dataset D3. The order of datasets according to the highest to the lowest precision of different prediction models is dataset D4, Dataset D3, dataset D2 and dataset D1. These observations of results shows that missing or unknown values adversely affect the precision of classifiers and when it was removed, dataset shows better result.

Performance of Diabetese Prediction Models based on different Dataset D1, D2, D3 and D4
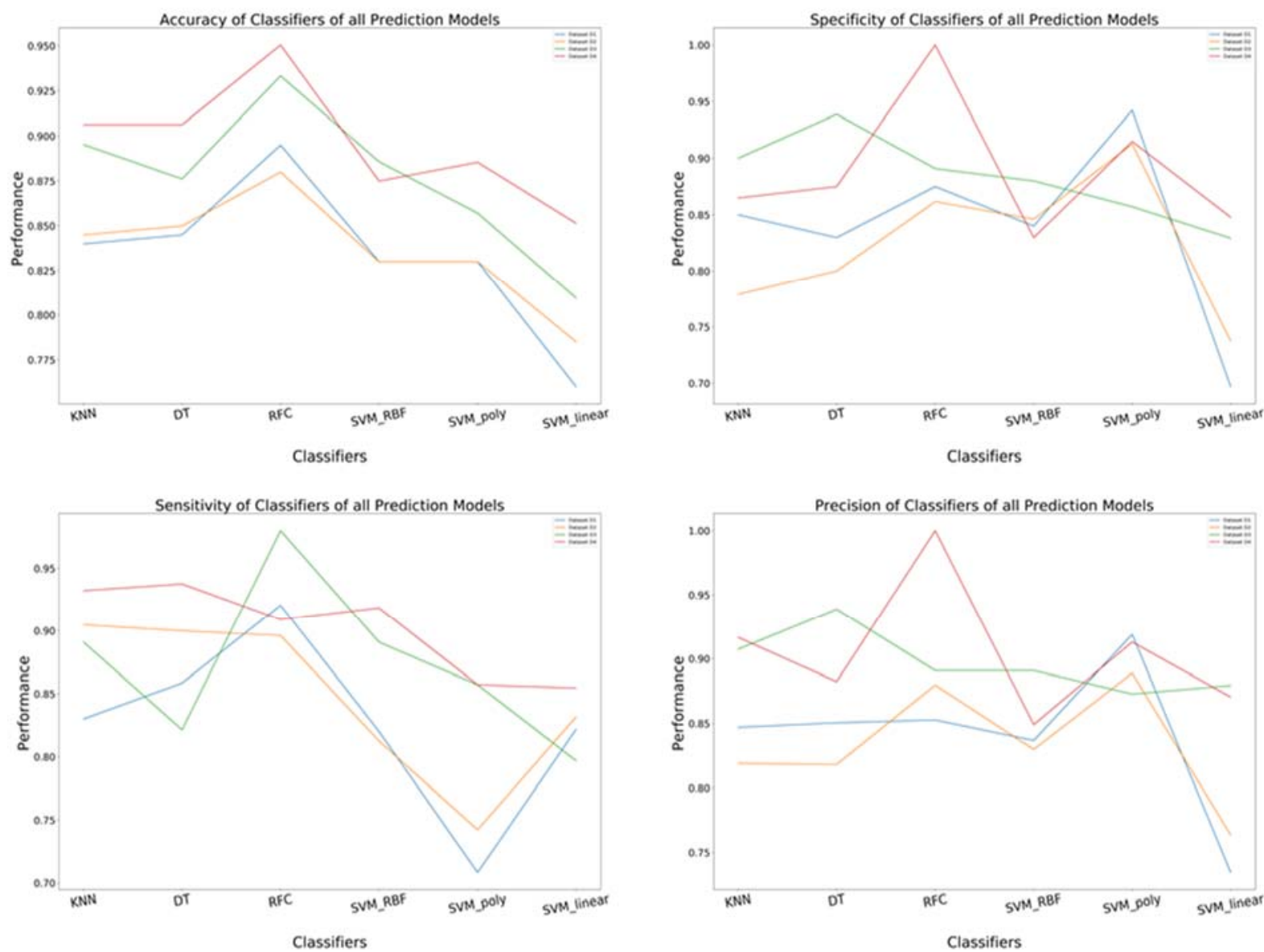

Fig 2: Accuracy, Specificity, sensitivity and Precision of classifiers of prediction models 


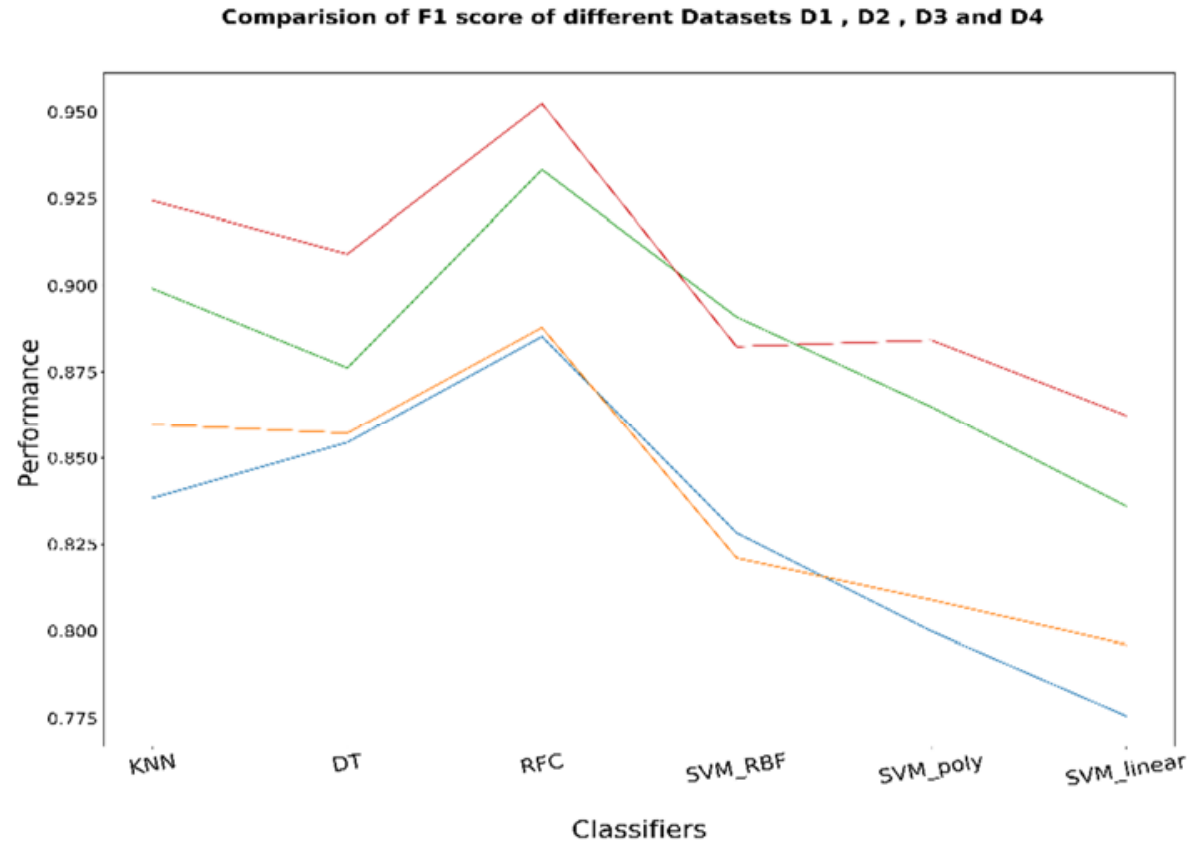

Fig 3- F1 Score of Classifiers for all dataset models

The maximum sensitivity is $98 \%$ achieved by random forest for D3 models, while the minimum is $70.83 \%$ for D1 models obtained from SVM classifier with polynomial kernel. For model on D4, the precision is $93.75 \%$ (by decision tree) and $92.05 \%$ by random forest for model on dataset D1.

The maximum specificity is $100 \%$, and it is achieved by random forest for model on D4, $94.23 \%$ by SVM for model on dataset D1, $91.26 \%$ by SVM for dataset D2 and $90.0 \%$ by KNN for dataset D3. It means they classify most of the negative samples correctly.

The figure Fig 2 shows that the maximum accuracy of classifiers of all models for dataset D1, D2, D3 and D4 is achieved by random forest, and it is $89.5 \%, 88.0 \%, 93.33 \%$ and $95.05 \%$ respectively. The best accuracy is achieved by model on dataset D4 and its performance is followed by models on D3, D2 and D1 dataset. From the observations, it proves that the preprocessing affects the performance of a classifier. From these a similar trend of accuracy is appears in datasets D1, D2 and D3 that SVM is the worst performing classifier.

The Fig-3 shows the F1 score of classifiers for all models. Among these model, the non- increasing order of models according to F1score achieved by classifiers, is D4, D3, D2 and D1. The model based on Dataset D1 shows the worst performance because dataset preprocessing was not done for it. An improvement is noticed in performance of models D2 in which missing values are replaced with column's mean values. Further, Model based on dataset D3 is better from model D2 since missing or unknown values are removed from dataset. And finally the best performance is shown by dataset model D4 in which missing values and outliers are removed from the dataset. From this, it may be concluded that the performances of classifiers are improved when input dataset is properly preprocessed. From observations, it is also cleared that random forest is the best performing classifier and SVM is the worst for all dataset models. Since dataset is balanced after oversampling of diabetes dataset, the macro and micro $\mathrm{fl}$ score of all classifiers are same.

The table- 4 , table-5, table- 6 , table- 7 and table- 8 show a detail views of results obtained from classifiers of prediction model on all dataset D1, D2, D3 and D4. According to a specific metric, each table shows the performance of classifiers of all prediction models and ranks them in the order of the best performing and the lowest performing classifiers. The performance of classifiers for dataset D4 is much better from the performance of dataset D1. From this, it may be concluded that the performances of classifiers are improved when missing or unknown values and outliers are removed from dataset with corresponding mean values while in dataset D3, removal of rows is performed from dataset which have missing or unknown values.

\subsection{Selection of the best Dataset Model for detail analysis}

The F1score and other metrics of different classification algorithms of models applied on these four datasets are given in Fig 4. Among these datasets, the prediction model based on dataset D4, in which outliers and rows having missing values are removed, shows the best performance. It shows the best F1score and accuracy of $95.24 \%$ and 
$95.05 \%$ respectively by Random forest classifier. The score of other performance metrics such as precision, sensitivity and specificity of model on this dataset is also better from other dataset models. Considering the performance of most of the classifiers for all datasets, the prediction model based on dataset D4 is the best model, and it is followed by dataset model D3, D2 and D1 respectively. Since this dataset shows the best accuracy, so we discuss other analytical features for it in detail.

Table 4. Comparative Analysis of Precision obtained by classifiers for different dataset models

\begin{tabular}{ccccccc}
\hline \multirow{2}{*}{$\begin{array}{c}\text { Prediction Models } \\
\text { based on different } \\
\text { Dataset }\end{array}$} & Max. (\%) & \multicolumn{2}{c}{ Classifier's Rank Arrange in Non-decreasing Order of Their Score } & Min. (\%) \\
\cline { 3 - 6 } & & Rank 1 & Rank 2 & Rank 3 & Rank 4 & \\
\hline Dataset Model D1 & 91.89 & SVM-Poly & RF & DT & KNN & 84.69 \\
Dataset Model D2 & 88.89 & SVM-Poly & RF & KNN & DT & 81.81 \\
Dataset Model D3 & 90.91 & DT & KNN & RF & SVM-Poly & 87.27 \\
Dataset Model D4 & 100 & RF & KNN & SVM-Poly & DT & 88.24 \\
\hline
\end{tabular}

Table 5. Comparative Analysis of Sensitivity obtained by classifiers for different dataset models

\begin{tabular}{lccccc}
\hline \multirow{2}{*}{$\begin{array}{c}\text { Prediction Models based } \\
\text { on different Dataset }\end{array}$} & $\begin{array}{c}\text { Max. } \\
(\mathbf{\%})\end{array}$ & \multicolumn{2}{c}{ Classifier's Rank Arrange in Non-decreasing Order of Their Score } \\
\cline { 3 - 6 } & & Rank 1 & Rank 2 & Rank 3 & Rank 4 \\
\hline Model on Dataset D1 & 92.05 & RF & DT & KNN & SVM-Poly \\
Model on Dataset D2 & 90.48 & KNN & DT & RF & SVM-Poly \\
Model on Dataset D3 & 98.0 & $R F$ & DT & KNN & SVM-Linear \\
Model on Dataset D4 & 93.75 & DT & KNN & SVM-RBF & RF \\
\hline
\end{tabular}

Table 6. Comparative Analysis of Specificity obtained by classifiers for different dataset models

\begin{tabular}{ccccccc}
\hline $\begin{array}{c}\text { Prediction Models } \\
\text { based on different } \\
\text { Dataset }\end{array}$ & Max. (\%) & \multicolumn{2}{l}{ Classifier's Rank Arrange in Non-decreasing Order of Their Score } & Min. (\%) \\
\cline { 3 - 5 } & & Rank 1 & Rank 2 & Rank 3 & Rank 4 & \\
\hline Dataset Model D1 & 94.23 & SVM-Poly & RF & KNN & DT & 82.98 \\
Dataset Model D2 & 91.26 & SVM-Poly & RF & DT & KNN & 77.89 \\
Dataset Model D3 & 90.00 & KNN & DT & RF & SVM-Linear & 82.93 \\
Dataset Model D4 & 100 & RF & SVM-Poly & DT & KNN & 86.49 \\
\hline
\end{tabular}




\section{Performance of Diabetese Prediction Models based on different Dataset D1, D2, D3 and D4}
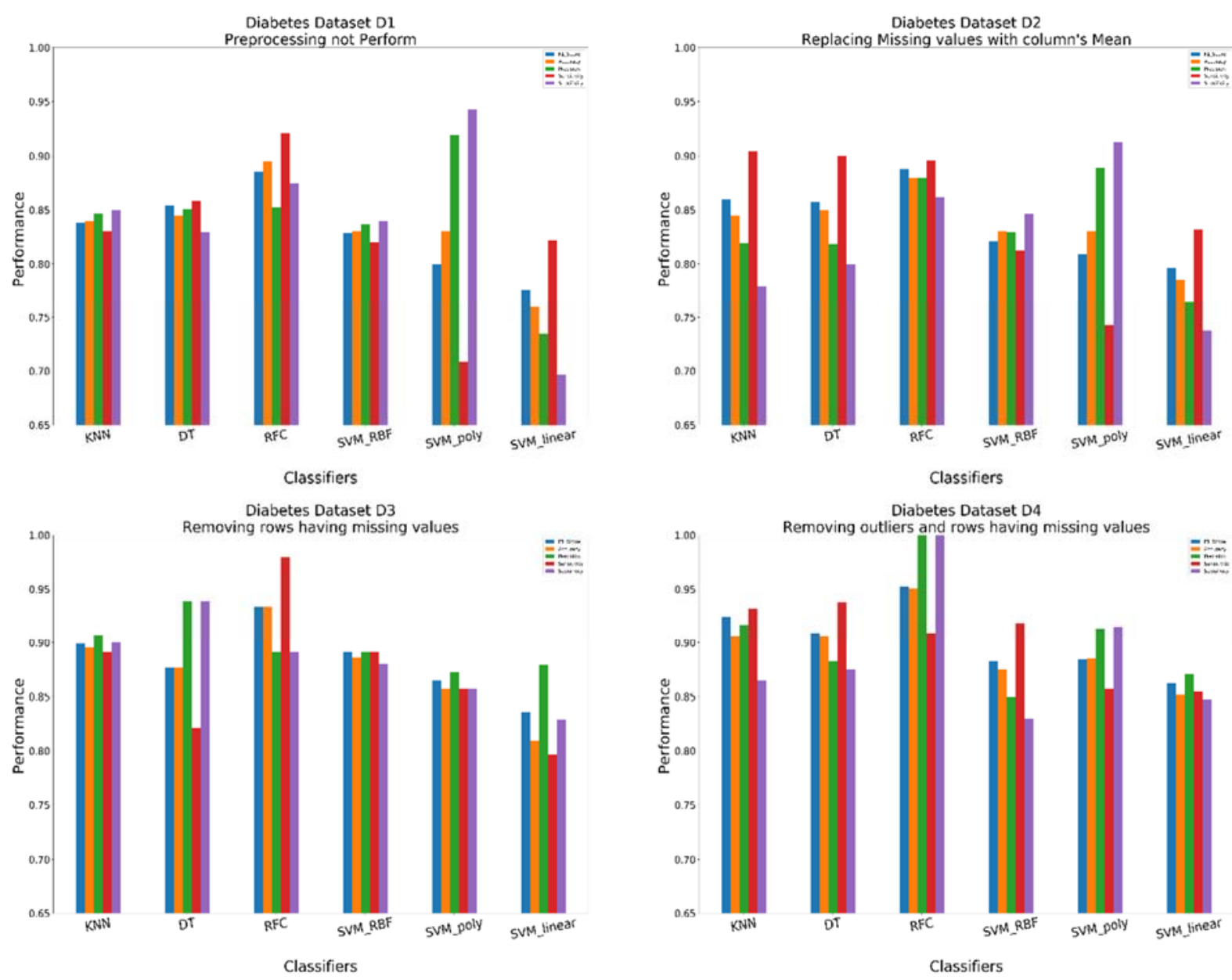

Fig 4- Performance of classifiers of prediction model on dataset D1, D2, D3 and D4

Table 7. Comparative Analysis of Accuracy obtained by classifiers for different dataset models

\begin{tabular}{lcccccc}
\hline \multirow{2}{*}{$\begin{array}{c}\text { Prediction Models } \\
\text { based on different } \\
\text { Dataset }\end{array}$} & Max. (\%) & \multicolumn{6}{c}{ Classifier's Rank Arrange in Non-decreasing Order of Their } \\
\cline { 3 - 6 } Score & Rank 1 & Rank 2 & Rank 3 & Rank 4 & Min. (\%) \\
\hline Dataset Model D1 & 89.5 & RF & DT & KNN & SVM-L & 76.0 \\
Dataset Model D2 & 88.00 & RF & DT & KNN & SVM-L & 78.5 \\
Dataset Model D3 & 93.33 & RF & DT & KNN & SVM-Linear & 80.95 \\
Dataset Model D4 & 95.05 & RF & DT & KNN & SVM-Linear & 85.15 \\
\hline
\end{tabular}

Table 8. Comparative Analysis of F1 Score obtained by classifiers for different dataset models

\begin{tabular}{lcccccc}
\hline \multirow{2}{*}{$\begin{array}{c}\text { Prediction Models based } \\
\text { on different Dataset }\end{array}$} & Max. (\%) & \multicolumn{2}{l}{ Classifier's Rank Arrange in Non-decreasing Order of Their Score } & \multirow{2}{*}{ Min. (\%) } \\
\cline { 3 - 6 } & & Rank 1 & Rank 2 & Rank 3 & Rank 4 & \\
\hline Dataset Model D1 & 88.52 & RF & DT & KNN & SVM-L & 77.57 \\
Dataset Model D2 & 88.79 & RF & KNN & DT & SVM-L & 79.62 \\
Dataset Model D3 & 93.33 & RF & DT & KNN & SVM-L & 83.61 \\
Dataset Model D4 & $\mathbf{9 5 . 2 4}$ & $\mathbf{R F}$ & KNN & $\boldsymbol{D T}$ & SVM-L & $\mathbf{8 6 . 2 4}$ \\
\hline
\end{tabular}




\section{Detail Analysis of Dataset Model D4}

The Dataset D4 is a subset of the original dataset and obtained by removing rows having outliers and missing values in any column. The shape of original dataset is $768 \mathrm{X} 9$ which is reduced in shape of $364 \mathrm{X} 9$ after dropping rows. It is an imbalanced dataset since it contains 112 positive samples and 252 negative samples. To make it balanced dataset, oversampling is done with SMOTE techniques and the dataset is reshaped in $504 \times 9$ containing 252 samples of each class. The model based on this dataset shows relatively better performance from other dataset models. So we make a detail analysis of performance of each classifier which made this dataset model best. Dataset D4 is divided in training and test dataset in 80:20 ratio, and classifier's hyperparameters are tuned to get better results.

\subsection{K- Nearest Neighbors Algorithm}

For the given dataset, K- Nearest Neighbors classifier is applied by taking the number of neighbors from 1 to 70 . The algorithm parameter for KNN classifier is 'auto' which means classifier attempts to determine the best approach from the training data by selecting a suitable algorithm from 'ball_tree', 'kd_tree', and 'brute' based on the values passed to fit method. The distance metric and leaf size of tree of classifier are \{'euclidean', 'minkowski\} and 30 respectively.

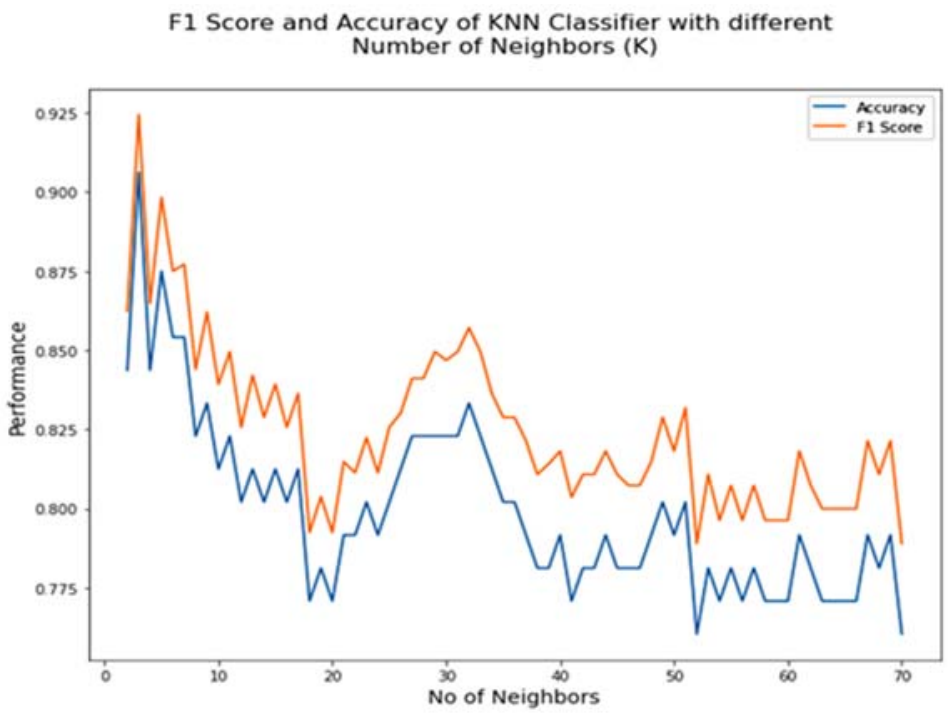

Fig 5: KNN classifier with different number of neighbors on dataset D4

Considering the graph (Fig 5) drawn between KNN classifier's performance and number of neighbors, it is found that accuracy of classifier is improved by increasing number of neighbors until performance touch its peak accuracy. Thereafter, increasing the number of neighbors does not improve its performance, although downs its performance. KNN classifier shows the best f1score of $92.44 \%$ when number of neighbors (K) is 3 . On these values of $\mathrm{K}$, the score of accuracy, precision, recall and specificity are $90.63 \%, 91.67 \%, 93.22 \%$ and $86.49 \%$ respectively.

\subsection{Decision Tree}

The input diabetic data set is a binary class label, so a standard decision tree classifier is suitable for it. The decision tree classifier has a number of hyperparameters and for better result a proper tuning of these values are required. The hyperparameters tuned to apply the decision tree classifier are information gain, maximum depth, minimum_sample_size and random state. It gets its best F1 score and accuracy when maximum_depth, minimum_sample_size, criterion are 12, 2 and 'entropy' respectively. The score of F1 score, accuracy, precision, sensitivity and specificity are $90.91 \%, 90.63 \%, 88.24 \%, 93.75 \%$ and $87.5 \%$ respectively.

\subsection{Random Forest}

It is an ensemble method that forms a number of decision trees for each class label. For dataset D4, it is the best scorer and its accuracy is $95.05 \%$, flscore $95.24 \%$, precision $100 \%$, recall $90.91 \%$ and specificity level is $100 \%$. The $100 \%$ precision indicates that all positive predictions made by classifier are actual positive. The performance of random forest depends upon a number of parameters such as minimum sample leaf size, N-estimators etc. The parameter 'N-estimators' of Random Forest classifier define the number of decision tree to form. From one dataset to another dataset, its value is varied to find the best result. For the given dataset, n_estimators' value has been 
tried between 1 and 50 , and it produces its best result when $n_{-}$estimators value is 41 . The same experiment is done with Min_sample_leaf value.

\subsection{Support Vector Machine}

SVM classifier uses four types of kernel to process data. These kernels are the linear kernel, the RBF kernel (Radial Basis Function Kernel), the polynomial kernel and the sigmoid kernel. In SVM classification, selection of kernel also plays an important role. We check the effect of RBF, polynomial and linear kernels and Regularized Parameter ' $\mathrm{C}$ ' for experimental analysis. The SVM classifier shows $88.54 \%$ accuracy level and $88.42 \% \mathrm{~F} 1$ score when kernel is 'poly' and regularized parameter is 1 . While the RBF kernel and linear kernel show less performance score in comparison to 'poly' kernel for the same dataset. The score of SVM classifier using different kernels are given in Table 9 -

Table 9. Performance of SVM Classifier on different hyper tuned Kernels and Regularized Parameter C

\begin{tabular}{lccc}
\hline SVM Kernel & $\begin{array}{c}\text { Radial Basis Function Kernel } \\
\text { (RBF) }\end{array}$ & Linear Kernel & Polynomial Kernel \\
\hline Regularized Parameter C & 47 & 83 & 1 \\
Accuracy & 87.5 & 85.15 & 88.54 \\
Precision & 84.1 & 87.04 & 91.3 \\
Sensitivity & 91.84 & 85.46 & 85.71 \\
Specificity & 82.98 & 84.78 & 91.49 \\
F1 Score & 88.24 & 86.24 & 88.42 \\
\hline
\end{tabular}

The table 10 shows the summarized view of performances of classifiers for prediction model based on dataset D4. The classifiers are ranked in the table according to their score of different metrics. It shows that random forest is the best performing classifier while SVM is the worst performer.

Table 10. Rank of classifiers for prediction Model based on dataset D4

\begin{tabular}{lllllll}
\hline \multirow{2}{*}{ Metrics } & \multirow{2}{*}{ Max. (\%) } & \multicolumn{4}{l}{ Classifier's Rank Arrange in Non-decreasing Order of Their Score } & Min. (\%) \\
\cline { 3 - 6 } & & Rank 1 & Rank 2 & Rank 3 & Rank 4 & 86.24 \\
\hline F1 Score & 95.24 & RF & KNN & DT & SVM- Linear & 85.15 \\
Accuracy & 95.05 & RF & DT & KNN & SVM- Linear & 84.91 \\
Precision & 100 & RF & KNN & DT & SVM- RBF & 85.46 \\
Recall & 93.75 & DT & KNN & RF & SVM-Linear & 82.98 \\
Specificity & 100 & RF & DT & KNN & SVM- RBF & \\
\hline
\end{tabular}

\section{Effect of Preprocessing: Performance Analysis from Dataset Points of View}

Dataset D1 and D2 are the same size and contain 768 samples. For both datasets model, the pattern of performance of classifiers are same. Random forest classifier shows the best performance amongst the performances of all classifiers for F1 score, accuracy and sensitivity metrics. F1 scores of this classifier for dataset D1 and dataset D2 are $88.52 \%$ and $88.79 \%$, accuracy is $89.50 \%$ and $88.0 \%$ and sensitivity is $92.05 \%$ and $89.62 \%$ respectively. SVM (Polynomial kernel) show the maximum precision and specificity for both dataset model. The performances of classifiers for dataset D2 is a little better from the performance of dataset D1. From this, it may be concluded that the performances of classifier for this dataset will be better when missing or unknown value is replaced with corresponding mean values.

For dataset D3, Random Forest Classifier is the best scorer in all categories of performance metrics. It shows maximum F1 score, accuracy and sensitivity. Its F1 Score and accuracy both are $93.33 \%$. The order of classifiers, arranged in decreasing order of their achieved F1score, is random forest, decision tree, KNN and SVM. Performance of dataset D3 is better from the performances of dataset model D1 and D2, which shows that when dataset is preprocessed and records having missing values are removed, performances of classifiers are improved.

Dataset D4 is created from original dataset by removing the samples which are either outliers or having missing values for some attributes. It has only 364 samples (after oversampling 504 samples) in number, which is the minimum in all four datasets. Random forest classifier is the best classifier for this dataset, showing maximum value for F1 Score, accuracy, precision and specificity. It shows maximum F1 score 95.24\%, accuracy $95.05 \%$, precision $100 \%$ and specificity $100 \%$. Its F1 Score is maximum in other dataset models for all classifiers. The $100 \%$ level of precision shows that all predictions which it declares as a positive result are actually positive. SVM is the worst performing classifier and shows minimum F1 score, accuracy, precision and sensitivity for this model. The classifier's performance for dataset model D4 is shown in figure-4. 
Since dataset D4 is created after removing samples which are either outliers or having missing values, the performance of prediction model based on D4 dataset is the best amongst all dataset models. It shows that classifiers show their best performance, when the input dataset is preprocessed properly, and it does not have noise such as outliers or samples having missing values. But there is some limitation with this dataset model. Performances may be affected for a dataset model in which most of the records having missing values. The table11 shows a tabulated representation of the best dataset prediction model and their performing classifier. It shows that the performance of D4 dataset model is best on most of the metrics and followed by D3 and D2 or D1. In all classifiers, Random Forest classifier works relatively better from other classifiers for all datasets. Random forest comprises a number of decision trees and select the predicted output by the majority votes. Random forest and decision tree beats other classifiers due to automatic feature interaction and less susceptibility to overfitting. KNN is a lazy learning model where the computations happen real time and take more time.

Table 11- Ranking of Prediction Models and theirs best performing classifiers

\begin{tabular}{lllllll}
\hline \multirow{2}{*}{ Metrics } & Max. (\%) & \multicolumn{4}{l}{$\begin{array}{l}\text { Ranking of datasets of Diabetes Prediction Model in non- increasing order } \\
\text { of their metrics }\end{array}$ (with best performing classifier) } & Min. (\%) \\
\cline { 3 - 6 } & & Rank 1 & Rank 2 & Rank 3 & Rank 4 & \\
\hline F1 Score & 95.24 & D4 (RF) & D3 (RF) & D2 (RF) & D1 (RF) & 88.52 \\
Accuracy & 95.05 & D4 (RF) & D3 (RF) & D1 (RF) & D2 (RF) & 88.00 \\
Precision & 100 & D4 (RF) & D1 (SVM-P) & D3 (DT) & D2 (SVM-P) & 88.89 \\
Recall & 98.00 & D3 (RF) & D4 (DT) & D1 (RF) & D2 (SVM) & 74.23 \\
Specificity & 100 & D4 (RF) & D1 (SVM-P) & D2 (SVM-P) & D3 (KNN) & 90.0 \\
\hline
\end{tabular}

\section{Findings of Analysis}

In this experiment there are four prediction models based on dataset D1, D2, D3 and D4 created from PIMA diabetes dataset using different preprocessing methods. The prediction model, based on dataset $\mathrm{D} 4$ that is created after the removing outliers and records having missing values, is the most robust model. Using random forest classifier, this model gives the highest score for F1 score, accuracy and other performance metrics. Performance of model based on dataset D3 is lower in comparison to prediction model on D4, but better than those of D2 and D1. From the analysis of these four versions of PIMA dataset, it is found that classifier's performances show variations by different preprocessing methods. From experimental analysis of these four prediction model, it is also found that Random Forest is the best classifier for all prediction model seeing f1score and accuracy.

Table 12 shows a detailed view of the best scoring classifier of each dataset from metrics points. From the analysis of the research, it is concluded that different preprocessing methods affect the performances of a classifier in different ways. The best performance is shown by PIMA diabetes prediction model based on D4 dataset, in which outliers and missing values records are removed during preprocessing. The same classifier shows different results when dataset is preprocessed in different levels. Random forest classifier shows different f1score for different dataset. For actual dataset D1, it shows lowest f1 score because it is not preprocessed. F1score is increased for D2 dataset when it is preprocessed by replacing missing values with the mean of corresponding columns. In D3 dataset, F1score increases to next level since samples having missing values are removed. While for dataset D4 it shows its best performance, even outliers are removed from dataset. It shows that classifiers do their best when input data is cleaned and noise free.

Table 12: Tabular Views of Performances of All Versions of PIMA Diabetes Datasets Showing the best performing Classifier

\begin{tabular}{|c|c|c|c|c|c|c|c|c|}
\hline \multirow[t]{2}{*}{$\begin{array}{l}\text { Performanc } \\
\text { e Metrics }\end{array}$} & \multicolumn{2}{|c|}{$\begin{array}{l}\text { Model on Dataset } \mathrm{D}_{1} \text { : } \\
\text { Actual PIMA Diabetes } \\
\text { Dataset }\end{array}$} & \multicolumn{2}{|c|}{$\begin{array}{l}\text { Model on Dataset } \mathrm{D}_{2}: \\
\text { Preprocessed as the } \\
\text { missing values are filled } \\
\text { by mean of columns }\end{array}$} & \multicolumn{2}{|c|}{$\begin{array}{l}\text { Model on Dataset } \mathrm{D}_{3} \text { : } \\
\text { Preprocessed as the } \\
\text { removal of rows } \\
\text { having missing values }\end{array}$} & \multicolumn{2}{|c|}{$\begin{array}{l}\text { Model on Dataset } D_{4}: \\
\text { Preprocessed as removal of } \\
\text { outliers and rows having } \\
\text { missing values }\end{array}$} \\
\hline & $\begin{array}{l}\text { Maximum } \\
\text { Score }\end{array}$ & $\begin{array}{l}\text { Best } \\
\text { Performe } \\
\mathrm{r}\end{array}$ & $\begin{array}{l}\text { Maximu } \\
\text { m Score }\end{array}$ & $\begin{array}{l}\text { Best } \\
\text { Performer }\end{array}$ & $\begin{array}{l}\text { Maxim } \\
\text { um } \\
\text { Score }\end{array}$ & $\begin{array}{l}\text { Best } \\
\text { Performer }\end{array}$ & $\begin{array}{l}\text { Maximum } \\
\text { Score }\end{array}$ & $\begin{array}{l}\text { Best } \\
\text { Performer }\end{array}$ \\
\hline F1 Score & 88.52 & $\mathrm{RF}$ & 88.79 & $\mathrm{RF}$ & 93.33 & RF & 95.24 & $\mathrm{RF}$ \\
\hline Accuracy & 89.5 & RF & 88.00 & RF & 93.33 & RF & 95.05 & $\mathrm{RF}$ \\
\hline Precision & 91.89 & SVM-P & 88.89 & SVM-P & 90.91 & DT & 100 & $\mathrm{RF}$ \\
\hline Recall & 92.05 & $\mathrm{RF}$ & 90.48 & KNN & 98.00 & $\mathrm{RF}$ & 93.75 & DT \\
\hline Specificity & 94.23 & SVM-P & 91.26 & SVM-P & 90.00 & KNN & 100 & RF \\
\hline
\end{tabular}




\section{Conclusion and Future Scope}

For a diabetes prediction system, diagnosis of diabetes in its early stage is very important for the cure of diabetic person. In this type of system, the cost of diagnosis of a diabetic person (true-positive) as a non-diabetic person (false-negative) is high. So the model should be so robust that it reduces these type errors. In this paper, to build a robust system, a single diabetic dataset is converted into four different datasets model D1, D2, D3 and D4 using different preprocessing methods. First model is based on D1 Dataset which use actual PIMA dataset, second model is on D2 dataset created after replacing the missing values with the mean of corresponding column, third model is based on dataset D3 created from removing the rows having missing values and the fourth model is based on the dataset obtained from removing outliers and samples having missing values. Since PIMA dataset is an imbalanced dataset, so oversampling of dataset is performed with SMOTE method to make balanced class dataset.

All prediction models use four machine learning classification techniques KNN, SVM, random forest and decision tree and analyze the performances to evaluate. Considering F1 score as the best metrics, the model based on dataset D4 is on the top of table. F1score, accuracy, precision and specificity of this model is the best from other model, and it is $95.24 \%, 95.05 \%, 100 \%$ and $100 \%$ respectively. Performance of D3 dataset model is a little bit lower from D4 dataset model but better than those of D2 and D1 dataset model. F1score, accuracy, precision, sensitivity and specificity of dataset model D3 are $93.33 \%, 93.33 \%, 90.1 \%, 98 \%$ and $90 \%$ respectively; for D2 dataset model it is $88.79 \%, 88.00 \%, 88.89 \%, 09.48 \%$ and $91.26 \%$ respectively and for model D1 it is $88.52 \%$, $89.5 \%, 91.89 \%, 92.05 \%$ and $94.23 \%$ respectively. From the analysis of the research, it is concluded that different preprocessing methods affect the performances of a classifier in different ways. The best performance is shown by prediction model based on D4 dataset, in which outliers and missing values records are removed during preprocessing.

Making the analysis of results obtained from different dataset models, it is concluded that outliers and missing values or unknown values present in a dataset may down the performances of classifiers. So for better prediction, it is necessary to remove those rows which have unknown or missing values. But it may create some problems for those datasets where most of the rows having missing or unknown values. If these rows are removed from the table, then the size of the dataset may reduce up to a critical level, and it might be difficult to make right prediction by model. The experimental analysis has been performed by a model on PIMA Indian Diabetes Dataset. The obtained observations are only for this dataset. In future, these observations can be tested by applying the model on other diabetes datasets. This analysis has been performed on small size dataset. To build a more robust model in future, large size datasets can be used with advance techniques such as deep learning algorithms. PIMA dataset is an imbalanced dataset, so the performance of prediction model can be observed using other metrics such as MCC, macro f1score and micro f1score.

\section{References}

[1] N. Sneha and T. Gangil, "Analysis of diabetes mellitus for early prediction using optimal features selection,” J. Big Data, vol. 6, no. 1, 2019, doi: 10.1186/s40537-019-0175-6.

[2] M. Alehegn, R. Joshi, and P. Mulay, "Analysis and Prediction of Diabetes Mellitus using Machine Learning Algorithm,” 2018.

[3] I. Kavakiotis, O. Tsave, A. Salifoglou, N. Maglaveras, I. Vlahavas, and I. Chouvarda, "Machine Learning and Data Mining Methods in Diabetes Research," Comput. Struct. Biotechnol. J., vol. 15, pp. 104-116, 2017, doi: 10.1016/j.csbj.2016.12.005.

[4] J. Steffi, R.Balasubramanian, and K. A. Kumar, "Predicting Diabetes Mellitus using Data Mining Techniques,” Int. J. Eng. Dev. Res., vol. 6, no. 2, pp. 460-467, 2018.

[5] V. V. Vijayan and C. Anjali, "Prediction and diagnosis of diabetes mellitus - A machine learning approach," 2015 IEEE Recent Adv. Intell. Comput. Syst. RAICS 2015, no. December, pp. 122-127, 2016, doi: 10.1109/RAICS.2015.7488400.

[6] World Health Organization, "Global Report on Diabetes," Geneva, 2016.

[7] A. Iyer, J. S, and R. Sumbaly, “Diagnosis of Diabetes Using Classification Mining Techniques,” Int. J. Data Min. Knowl. Manag. Process, vol. 5, no. 1, pp. 01-14, 2015, doi: 10.5121/ijdkp.2015.5101.

[8] A. D. Association, "Classification and diagnosis of diabetes: Standards of medical care in Diabetesd2018," Diabetes Care, vol. 41, no. Supplement 1, pp. S13-S27, Jan. 2018, doi: 10.2337/dc18-S002.

[9] M. Thirugnanam, P. Kumar, S. Vignesh Srivatsan, and C. R. Nerlesh, "Improving, the prediction rate of diabetes diagnosis using fuzzy, neural network, case based (FNC) approach,” Procedia Eng., vol. 38, pp. 1709-1718, 2012, doi: 10.1016/j.proeng.2012.06.208.

[10] R. Sengamuthu, R. Abirami, and D. Karthik, "Various Data Mining Techniques Analysis To Predict," Int. Res. J. Eng. Technol., vol. 5 , no. 5, pp. 676-679, 2018.

[11] "Diagnostic Criteria and Classification of Hyperglycaemia First Detected in Pregnancy,” WHO, pp. 1-63, 2013

[12] T. Daghistani and R. Alshammari, "Diagnosis of Diabetes by Applying Data Mining Classification Techniques," Int. J. Adv. Comput. Sci. Appl., vol. 7, no. 7, 2016, doi: 10.14569/ijacsa.2016.070747.

[13] A. Azrar, Y. Ali, M. Awais, and K. Zaheer, "Data mining models comparison for diabetes prediction," Int. J. Adv. Comput. Sci. Appl., vol. 9, no. 8, pp. 320-323, 2018, doi: 10.14569/ijacsa.2018.090841.

[14] F. Mercaldo, V. Nardone, and A. Santone, "Diabetes Mellitus Affected Patients Classification and Diagnosis through Machine Learning Techniques," Procedia Comput. Sci., vol. 112, pp. 2519-2528, 2017, doi: 10.1016/j.procs.2017.08.193.

[15] N. Nai-Arun and R. Moungmai, "Comparison of Classifiers for the Risk of Diabetes Prediction," Procedia Comput. Sci., vol. 69, pp. 132-142, 2015, doi: 10.1016/j.procs.2015.10.014.

[16] P. Zhang, C. Fonnesbeck, D. C. Schmidt, J. White, and S. A. Mulvaney, "Understanding Barriers to Diabetes Self-Management Using Momentary Assessment and Machine Learning," pp. 1-21.

[17] "Cloud-based diabetes coaching platform for diabetes management - IEEE Conference Publication." [Online]. Available: https://ieeexplore.ieee.org/document/7455972/;jsessionid=oggGDVogPcPXiQFJCyH9kmKOtYiwen5Vn64hLiYCruJokMj9_gS9! 
1796626962. [Accessed: 08-Oct-2020].

[18] P. S. Kumar and V. Umatejaswi, "Diagnosing Diabetes using Data Mining Techniques," International Journal of Scientific and Research Publications, 2017. [Online]. Available: http://www.ijsrp.org/research-paper-0617.php?rp=P666543. [Accessed: 26Nov-2020].

[19] S. Hina, A. Shaikh, and A. Sattar, “Analyzing Diabetes Datasets using Data Mining,” J. Basic Appl. Sci., vol. 13, pp. 466-471, 2017, doi: 10.6000/1927-5129.2017.13.77.

[20] R. B. Lukmanto and E. Irwansyah, "The Early Detection of Diabetes Mellitus (DM) Using Fuzzy Hierarchical Model," Procedia Comput. Sci., vol. 59, no. Iccsci, pp. 312-319, 2015, doi: 10.1016/j.procs.2015.07.571.

[21] K. Maladkar, "5 Ways To Handle Missing Values In Machine Learning Datasets," 2018. [Online]. Available: https://analyticsindiamag.com/5-ways-handle-missing-values-machine-learning-datasets/. [Accessed: 14-Nov-2020].

[22] R. Dwivedi, "What is Imblearn Technique - Everything To Know For Class Imbalance Issues In Machine Learning," 2020. [Online]. Available: https://analyticsindiamag.com/what-is-imblearn-technique-everything-to-know-for-class-imbalance-issues-inmachine-learning/. [Accessed: 08-Nov-2020].

[23] J. Brownlee, "SMOTE for Imbalanced Classification with Python," 2020. [Online]. Available: https://machinelearningmastery.com/smote-oversampling-for-imbalanced-classification/. [Accessed: 15-Nov-2020].

[24] N. V. Chawla, K. W. Bowyer, L. O. Hall, and W. P. Kegelmeyer, "SMOTE: Synthetic Minority Over-sampling Techniques," J. Artif. Intell. Res., vol. 16, no. 2, pp. 321-357, 2002.

[25] T. Santhanam and M. S. Padmavathi, "Application of K-Means and genetic algorithms for dimension reduction by integrating SVM for diabetes diagnosis,” Procedia Comput. Sci., vol. 47, no. C, pp. 76-83, 2015, doi: 10.1016/j.procs.2015.03.185.

[26] S. C. Gupta and N. Goel, "Performance enhancement of diabetes prediction by finding optimum K for KNN classifier with feature selection method," pp. 980-986, 2020, doi: 10.1109/icssit48917.2020.9214129.

[27] A. Jakka and V. R. J, "Performance Evaluation of Machine Learning Models for Diabetes Prediction," Int. Journalof Innov. Technol. Exloring Eng., vol. 8, no. 11, pp. 1976-1980, 2019, doi: 10.35940/ijitee.K2155.0981119.

[28] M. Renuka Devi and J. Maria Shyla, “Analysis of various data mining techniques to predict diabetes mellitus," Int. J. Appl. Eng. Res., vol. 11, no. 1, pp. 727-730, 2016.

[29] D. Dua and C. Graff, "UCI Machine Learning Repository," CA: University of California, School of Information and Computer Science. [Online]. Available: http://archive.ics.uci.edu/ml.

\section{Authors Profile}
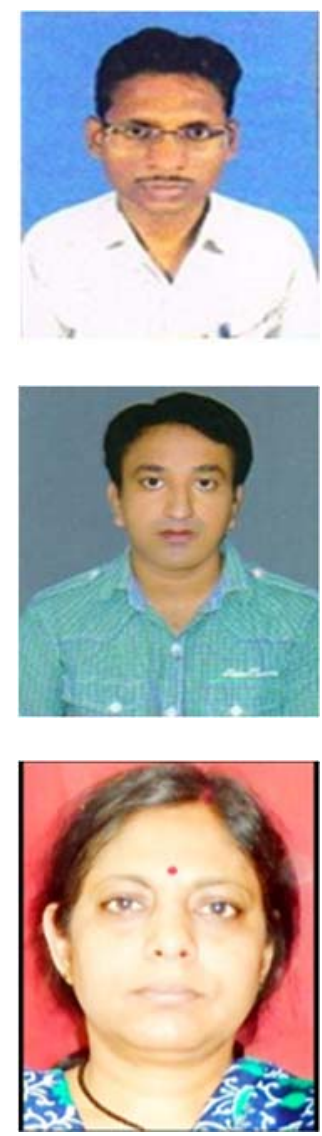

Subhash Chandra Gupta is a research scholar in the Department of Computer Applications of V.B.S. Purvanchal University, Jaunpur. He has received his M.C.A. degree from Indira Gandhi National Open University, New Delhi. His research area is Data mining. He has published some articles in international journals and conferences. His interests include data mining, python, data structure and algorithms.

Durgesh Kumar Singh is Research Scholar in the Department of Computer Applications of Veer Bahadur Singh Purvanchal University, Jaunpur. He has received her M.C.A. degree in computer applications from Dr. K. N Modi Institute of Engineering \& Technology, Ghaziabad. His research area is customer relationship management using data mining technology, and has published some review articles in international journals and conferences and participated in many workshop. His interests include data mining, python, $\mathrm{R}$ programming languages, Java.

Dr. Noopur Goel is currently working as an Assistant Professor in the Department of Computer Applications, Veer Bahadur Singh Purvanchal University, Jaunpur, and have more than 20 years of teaching experience. She has achieved her Ph.D. (Computer Science) from Banaras Hindu University, Varanasi. Her research interests include Software Testing, Software Reuse. She is serving as an editorial member and reviewer of several international reputed journals. Dr. Noopur Goel is the member of many international affiliations. She has successfully completed her Administrative responsibilities. She has authored of many research articles/books related to Software Testing, Software Reuse. 